\title{
Top hat electrostatic analyzer for far-field electric propulsion plume diagnostics
}

\author{
Allen L. Victor, ${ }^{\text {a) }}$ Thomas H. Zurbuchen, and Alec D. Gallimore \\ Plasmadynamics and Electric Propulsion Laboratory, Department of Aerospace Engineering, \\ University of Michigan, Ann Arbor, Michigan 48109
}

(Received 29 June 2005; accepted 14 December 2005; published online 25 January 2006)

\begin{abstract}
The design, development, and testing of the top hat electric propulsion plume analyzer (TOPAZ) are presented for far-field electric propulsion plume diagnostics. The trend towards high-power thruster development will require plume diagnostic techniques capable of measuring high-energy particles as well as low-energy ions produced from charge-exchange collisions due to elevated facility background pressures. TOPAZ incorporates a "top hat" design with a geometrical analyzer constant of 100 resulting in a wide energy range and a high-energy resolution. SIMION, an ion trajectory analysis program, was used to predict characteristics of the analyzer. An ion beam accelerator system confirms the computational results. TOPAZ provides an energy resolution of $2.7 \%$, field of view of $112^{\circ} \times 26^{\circ}$ (azimuthal by elevation) with an angular resolution in each direction of $2^{\circ}$, and a demonstrated energy-per-charge acceptance range of 5-15 keV. An energy profile measurement of the NASA-173Mv1 Hall thruster demonstrates instrument operation in a Hall thruster plume. (C) 2006 American Institute of Physics. [DOI: 10.1063/1.2165549]
\end{abstract}

\section{INTRODUCTION}

Electric propulsion (EP) offers power-efficient, high specific impulse $\left(I_{\mathrm{sp}}\right)$ options for deep-space missions as well as station keeping, orbital transfer, and attitude control requirements for near-Earth spacecraft. Hall thrusters are a type of EP system that utilizes electric and magnetic fields to produce thrust. Electrons emitted by a cathode travel upstream towards a positively charged anode. A magnetic field that is applied in the perpendicular direction of the electric field hinders electron motion and creates a closed electron drift region. Propellant (e.g., xenon or krypton) is injected at the anode of an annular discharge channel and ionized through collisions with the electrons caught in the closed electron drift region. The magnetic field has very little effect on the relatively massive ions. The electric field, however, accelerates the ions downstream away from the anode producing thrust.

Recent trends in Hall thruster research by the USAF and US industry have included the high-power $(>20 \mathrm{~kW})$ regime. NASA is sponsoring high-power/high- $I_{\mathrm{sp}}(50 \mathrm{~kW} />$ $=4000 \mathrm{~s})$ Hall thruster technology through the NASA Glenn Research Center. ${ }^{1}$ The NASA-457 Hall thruster developed at GRC produced the highest-power level and thrust $(75 \mathrm{~kW}$ and $2.9 \mathrm{~N}$ ) achieved by any Hall thruster. ${ }^{2}$ High- $I_{\mathrm{sp}}$ anodelayer-type (TAL) Hall thrusters have achieved specific impulses above $4100 \mathrm{~s}$ at this center as well. ${ }^{3}$

For these high-powered engines and future even more powerful engines being developed, plume characterization is imperative for determining their effect on spacecraft systems. Plasma transport properties, ionic charge state, and ion en-

\footnotetext{
${ }^{a)}$ Electronic mail: victora@umich.edu
}

ergy distributions are also important for understanding how Hall thrusters work and for improving their performance. ${ }^{4}$

One technique for determining the energy-per-charge distribution of positively charged ions in the plasma is to use an electrostatic analyzer. A specific geometry for the electrostatic analyzer, which allows for a wide field of view, is the top hat analyzer. This electrostatic analyzer consists of two concentric hemispheres, with an aperture at the apex of the outer hemisphere (see Fig. 1). The inner hemisphere is biased to a negative voltage while the outer hemisphere is grounded. The electric field created between the plates allows for particles within a specific energy range (corresponding to the inner plate voltage) to pass through the aperture and through the gap between the plates. By virtue of its geometry, the top hat analyzer is capable of having a $360^{\circ}$ azimuthal field of view. Steering electric fields created by a guiding plate and top hat plate above the aperture allow for a field of view in the vertical direction as well. Structural constraints, however, diminish the total field of view in both directions.

The motivation for the design of the top hat electric propulsion plume analyzer (TOPAZ) is first discussed. The analytical and Monte Carlo design of TOPAZ is then described. Characterization and performance measurements conducted through the use of an ion beam and Hall thruster are presented as well.

Although a similarity in name exists, there is no relationship between this instrument and the Russian nuclear reactors developed for space applications in the 1960s.

\section{DESIGN MOTIVATION}

Electrostatic analyzers have been and are currently employed on spacecraft to investigate space plasmas such as the solar wind as well as the ionospheres and magnetospheres of 


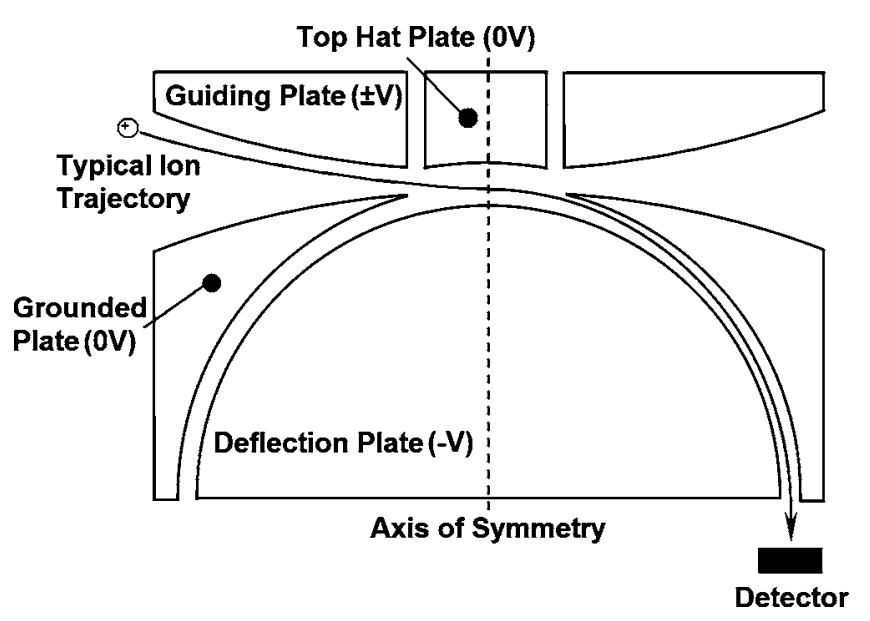

FIG. 1. Major components and a typical ion trajectory for a top hat electrostatic analyzer.

Earth and other planets. ${ }^{5,6}$ Space plasmas offer a wide range of particle energies from less than $1 \mathrm{eV}$ to several MeV. The desire to study these particles has led to the design of electrostatic analyzers capable of detecting particles over several orders of magnitude in energy ${ }^{5}$ however, these types of plasmas have an ion number density several orders of magnitude lower than Hall thruster and ion engine plumes. Figure 2 describes the typical energy and number density ranges of space, laboratory, and Hall thruster and ion engine plasmas. The Hall thruster plume, the plasma of interest for TOPAZ, is nestled between laboratory plasmas (thetatrons and fusion reactors) and space plasmas (solar wind and the magnetotail) on the density scale and at the magnetotail plasma in terms of energy.

There are many examples of space plasma detectors that use the top hat configuration. EP plume measurements with this type of device, however, are much rarer. The plasma experiment for planetary exploration (PEPE), flown on Deep Space 1 (DS1), ${ }^{7}$ included a duel top hat analyzer to measure electrons and ions from the solar wind, the spacecraft photoelectron sheath, and products of the xenon-ion propulsion system (XIPS). Low-energy xenon ions at $\sim 20 \mathrm{eV}$ created from the beam ion interaction with neutral xenon particles leaving the thruster were observed by PEPE. ${ }^{8}$

Although beam ions were not measured by PEPE due to

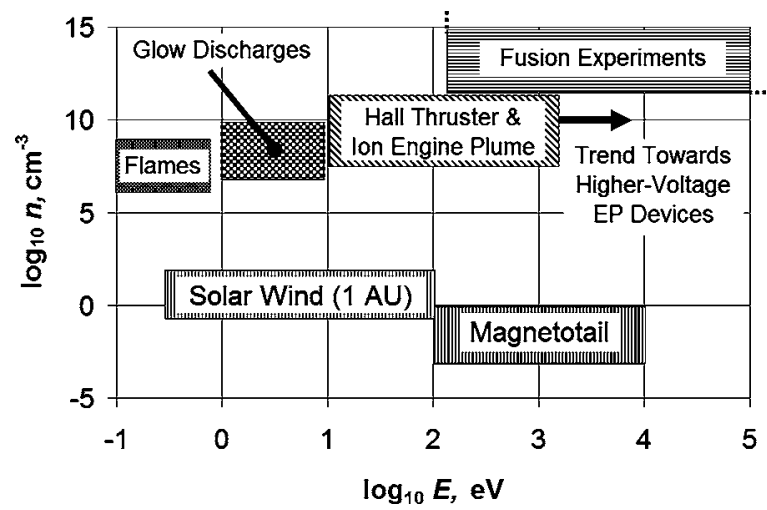

FIG. 2. Number density and energy of typical space, laboratory, and electric propulsion plasmas. the position and orientation of the thruster with respect to the instrument, the observation of charge-exchange ions provides evidence that the top hat analyzer can be used as a plume diagnostics tool for characterizing facility effects. Hall thrusters have been shown to yield higher current-density profiles in the far-field plume at higher background pressures. It is theorized that charge-exchange ions created from beam ions and neutral background particles are the culprit for the elevated current densities. ${ }^{9}$ Diagnostic tools capable of characterizing the low-energy charge-exchange ions as well as the high-energy beam ions are therefore necessary to distinguish facility effects on plume diagnostics.

\section{ANALYZER DESIGN}

The TOPAZ design process is discussed in the following section. The design requirements for the analyzer are first described. A derivation of the ideal analyzer geometry is then presented. SIMION, ${ }^{10}$ an ion trajectory code, was used to predict the performance (e.g., resolutions) of TOPAZ. The final design of the analyzer was determined through an iterative process with SIMION and the additional structural constraints modeled in the CAD program PRO/ENGINEER. ${ }^{11}$

\section{A. Design requirements}

An increase in power is expected for EP thruster development, and the acceleration potentials (and hence beam ion energies) are expected to increase in the foreseeable future. While Hall thruster voltages are not expected to increase above $2 \mathrm{kV}$, ion thrusters that are being considered for deepspace missions may have xenon-ion beams of energy above $6 \mathrm{keV}^{12} \mathrm{~A} \mathrm{Xe}^{2+}$ ion would accelerate to $12-14 \mathrm{keV}$ at this potential; hence TOPAZ was designed to have a high-energy measurement capability up to $15 \mathrm{keV}$. The nature of a top hat analyzer allows for the lower bound to be close to $0 \mathrm{eV}$, since the plate potentials correspond directly with the measured energy. The lower-energy bound therefore is set by the accuracy of the power supplies used.

Since TOPAZ is a far-field plume diagnostics instrument, an adequate field of view of the thruster is required to "image" the ions projected from the entire discharge channel. A $30^{\circ}$ vertical field of view allows for $54 \mathrm{~cm}$ of an object to be viewed from $1 \mathrm{~m}$ away, well within the size range of most thrusters. The azimuthal field of view is ideally $360^{\circ}$, but structural constraints diminish this to $112^{\circ}$. The angular resolutions of the field of view were expected to be $2^{\circ} \times 2^{\circ}$ for the vertical and azimuthal directions, respectively. This resolution provides enough accuracy to determine a detected ion from the discharge channel, a different part of the thruster, or from the plume.

\section{B. Theory of operation}

The top hat analyzer utilizes a radial electric field to guide ions through a spherical shell-shaped channel between a grounded plate and a negatively charged deflection plate. Figure 3 displays the key dimensions and plates for a top hat analyzer. The most important criterion is the ratio of the channel radius $R_{C}$ to the gap distance $\Delta R\left(\Delta R=R_{2}-R_{1}\right)$ 


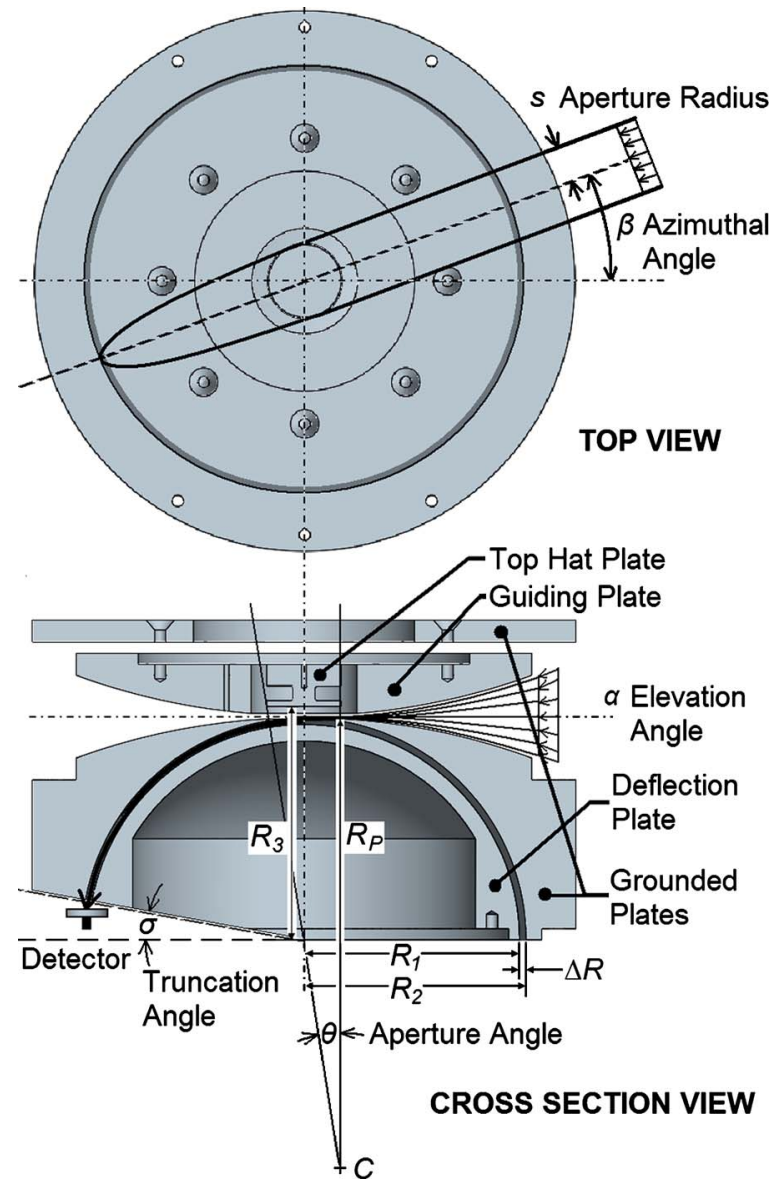

FIG. 3. (Color online) Principal design parameters of a top hat analyzer.

which sets the analyzer constant [Eq. (1)]. The channel radius $R_{C}$ is simply the average of the inner and outer radii for the gap:

$$
K \equiv \frac{R_{C}}{\Delta R} .
$$

The analyzer constant $K$ determines the energy resolution, energy-to-voltage ratio, and other properties of the analyzer. Equating the applied force required to turn a particle at the channel radius with the electric field generated in the gap, the voltage difference between the spherical surfaces is related to the energy per charge. For high analyzer constants, the electric field can be assumed to be uniform between the deflection plate and grounded plate. Equation (2) displays the simple relationship between the deflection plate voltage $V_{D}$, the analyzer constant, and the expected energy per charge $E / q$ (in units of $\mathrm{J} /$ electron charge) to be measured:

$$
\frac{E}{q}=-\frac{K V_{D}}{2} .
$$

The inner deflection plate radius $R_{1}$ is held to a negative plate potential $V_{D}$ to detect positively charged ions, while the outer radius is kept at ground potential. The top hat plate is also usually held at ground, but can be biased to increase transmissivity if needed. A maximum ratio of detectable ions to incoming ions is realized when the top hat radius $R_{3}$ is larger than the deflection plate radius $R_{1}$ by twice the gap distance (i.e., $R_{3}=R_{1}+2 \Delta R$ ). ${ }^{13}$ This distance yields an elec- tric field over the aperture that is half that of the electric field in the channel. Hence, the particle travels with a radius $R_{p}$ (with the center marked as point $C$ in Fig. 3) of approximately twice the channel radius over the aperture between the top hat plate and the deflection plate.

Guiding plates, which can vary either positively or negatively in plate potential, allow for variance in the vertical angular direction (elevation angle) for the measured ions. Ions coming from the selected elevation angle are guided into the top hat region with a horizontal trajectory. The guiding plate radius of curvature is determined by setting the outer entrance angle of the surface slightly higher than the desired elevation angle $\alpha$ field of view. This angle is mirrored on the lower grounded plate surface. Through simple trigonometry the guiding plate radius $R_{G}$ is related to the top hat plate radius and the entrance surface angle in Eq. (3):

$$
R_{G}=\frac{R}{\sin \alpha} \text {. }
$$

Particles enter the top hat aperture and are turned at a radius $R_{P}$ due to the electric field generated between $R_{3}$ and $R_{1}$. As mentioned previously, the electric field is approximately half that of the gap; therefore, $R_{P}$ is approximately $2 R_{2}$. The center of curvature for the particles is at point $C$ (see Fig. 3). To determine the optimum aperture angle $\theta$, the "grazing" trajectory of a particle is followed that touches the front lip of the top hat plate and follows the outer radius of the gap. The particle travels at a radius of $2 R_{2}$ due to the field generated between the top hat plate and the deflection plate. Through the construction of a right triangle between point $C$ and the top hat entrance lip and the outer gap radius, the optimum aperture angle $\theta$ can be derived [Eq. (4)]:

$$
\cos \theta=\frac{2 R_{2}-\Delta}{R_{P}} .
$$

Since $R_{P} \approx 2 R_{2}$ and $R_{C} \approx R_{2}$ the aperture angle can be rewritten as a function of the analyzer constant through Eq. (1).

$$
\cos \theta \approx 1-\frac{1}{2 K} \text {. }
$$

Through a two-term Taylor expansion of the cosine function, the aperture angle is directly correlated with the analyzer constant for $\theta<15^{\circ}$ :

$$
\cos \theta=1-\frac{1}{2} \theta^{2} \cdots \text {. }
$$

Therefore, the aperture angle (in radians) is proportional to the inverse square root of the analyzer constant:

$$
\theta \approx \sqrt{\frac{1}{K}}
$$

The optimum truncation angle $\sigma$ for ion focusing at the detector is $\sim \theta / 2 .{ }^{13}$ As the analyzer constant is increased (above 50), this value becomes small and for TOPAZ is negligible and therefore is ignored. Ions entering over the aperture diameter $2 s$ are focused at the exit of the gap while maintaining their entrance azimuthal angle $\beta$ (shown in the top view of Fig. 3). 
The above formulation provided the general basis for an analytic attack on the design of TOPAZ to meet the design requirements. Only an approximate response can be estimated, since nonlinear surfaces and fringe effects from structural constraints are difficult to model analytically. SIMION, an ion optics program, allowed for a more detailed design and characterization of TOPAZ to be determined.

\section{Design of TOPAZ through SIMION}

SIMION is a computer code that is capable of modeling ion optics with electrostatic and/or magnetic potential arrays. For the purposes of TOPAZ, only electrostatic fields were modeled. First, a model of TOPAZ was defined through a geometry file that included the volume definitions and potentials of the instrument. TOPAZ was assumed to be cylindrically symmetric through the eyes of SIMION. Then the electric potential $\varphi$ was solved for around the instrument through the Laplace equation [Eq. (8)]. SIMION assumes a zero charge volume density (no space charge) $:^{10}$

$$
\nabla^{2} \phi=0 .
$$

Over-relaxation, a finite difference technique, was used as the iterative process to converge on the electric potential field solution. After the potential field was determined, ion trajectories were modeled by determining the electrostatic acceleration on the particle. SIMION incorporates a standard fourth-order Runge-Kutta method for integrating out the ion's trajectory. ${ }^{13}$

In determining the optimal design for TOPAZ, several configurations with differing analyzer constants were simulated. Over $1 \times 10^{6}$ ion trajectories were simulated to "fly" into the instrument to determine the instrument's response for each configuration. An analyzer constant above 55 was found to be sufficient to provide an energy resolution of better than 5\%. Beyond analyzer constants of 100, the energy resolution does not change appreciably, and manufacturing considerations become important, since an exceedingly small gap must exist over a large radius. A high analyzer constant was chosen, since TOPAZ would be constructed on a highly accurate computer numerical controlled (CNC) lathe. This high analyzer constant allows for the energy resolution requirement of $5 \%$ to be easily met at $2.2 \%$. A channel radius of $10 \mathrm{~cm}$ was chosen, requiring a gap distance of $1 \mathrm{~mm}$ to yield an analyzer constant of 100 . Also, a small gap distance is ideal for the Hall thruster plume, since space-charge effects from the high-density plasma could interfere with the electric field generated by a gap that was too large.

For an ideal top hat with $K=100$, Eq. (7) yields an aperture angle $\theta$ of $5.72^{\circ}$. The top hat radius $R_{3}$ would need to be held $2 \mathrm{~mm}$ from the deflection plate over this angle. This requirement was difficult to achieve, since the thickness of the lip of grounded plate at the gap entrance was large with respect to this distance. The top hat plate would be precariously close to the grounded plate. To alleviate this dilemma, the top hat plate was raised further away from the deflection plate to $3.5 \mathrm{~mm}$. To maintain the optimum transmissivity the top hat plate must be biased positive with respect to the negatively biased deflection plate. The transmission of ions from the entrance of TOPAZ into the aperture could be in-
TABLE I. Physical characteristics of TOPAZ.

\begin{tabular}{lc}
\hline \multicolumn{1}{c}{ Parameter } & \multicolumn{1}{c}{ Value } \\
\hline Analyzer constant $K$ & 100 \\
Inner gap radius $R_{1}$ & $9.95 \mathrm{~cm}$ \\
Outer gap radius $R_{2}$ & $10.05 \mathrm{~cm}$ \\
Top hat plate distance & $10.30 \mathrm{~cm}$ \\
$\quad$ (along centerline) $R_{3}$ & \\
Gap distance $\Delta R$ & $1.0 \mathrm{~mm}$ \\
Instrument size (diameter) & $24.6 \mathrm{~cm}$ \\
Guiding plate radius $R_{G}$ & $30.1 \mathrm{~cm}$ \\
Aperture angle $\theta$ & $9.60^{\circ}$ \\
Aperture radius $s$ & $1.70 \mathrm{~cm}$ \\
Geometric factor $G(E)($ at $1 \mathrm{keV})$ & $2.4 \times 10^{-5} \mathrm{~cm}^{2} \mathrm{sreV} / \mathrm{eV}$ \\
\hline
\end{tabular}

creased by biasing the top hat plate to a positive voltage.

The aperture radius $s$ was increased to $1.70 \mathrm{~cm}$, providing an aperture angle of $\theta=9.60^{\circ}$ (see Fig. 3). The combination of increasing the aperture size and moving the top hat plate away from the deflection plate prevents the guiding plate, top hat plate, and deflection plate from interfering with each other through unwanted electric fields.

At the entrance of the instrument, the guiding plate and grounding plate have surface angles of $\pm 20^{\circ}$ with respect to the horizontal symmetry line between these plates (see Fig. 3 ). This provides TOPAZ with an elevation angular field of view of approximately $\pm 15^{\circ}$. Through Eq. (3), the guiding plate radius required to produce this was $30.1 \mathrm{~cm}$.

The geometric factor $G(E)$ (not to be confused with the geometrical analyzer constant) describes the overall angular, energy, and acceptance area for the instrument. This value was calculated via SIMION by finding the ratio of detected ions to flown ions and multiplying this fraction by the ranges of parameters varied. Specifically, the ranges for the elevation and azimuthal angles $\Delta \alpha$ and $\Delta \beta$, horizontal and vertical starting positions $\Delta x$ and $\Delta y$, and energies $\Delta E$ of the virtual particles are varied. This product is described in Eq. (9) and was calculated from a $1 \mathrm{keV}$ beam and normalized by the average energy of the particles $E_{\mathrm{av}}$ :

$$
G(E)=\left(\frac{\text { No. of detected ions }}{\text { Total flown ions }}\right) \frac{\Delta E}{E_{\mathrm{av}}} \Delta \alpha \Delta \beta \Delta x \Delta y .
$$

Angular resolutions, the elevation angle field of view, and the plate voltage-energy relationship could be accurately determined through SIMION. The computational results are discussed in conjunction with the experimental calibration measurements for comparison in the Analyzer Calibration section of this article. The design specifications discussed above are summarized in Table I.

\section{Operational considerations}

Since TOPAZ is designed to be placed in front of a highenergy and high-density ion environment, precautions must be taken to protect the instrument from erosion due to sputtering damage. A protective box enclosure composed of graphite has been designed to shield TOPAZ from the highenergy incident-beam ions emanating from the thruster. The 


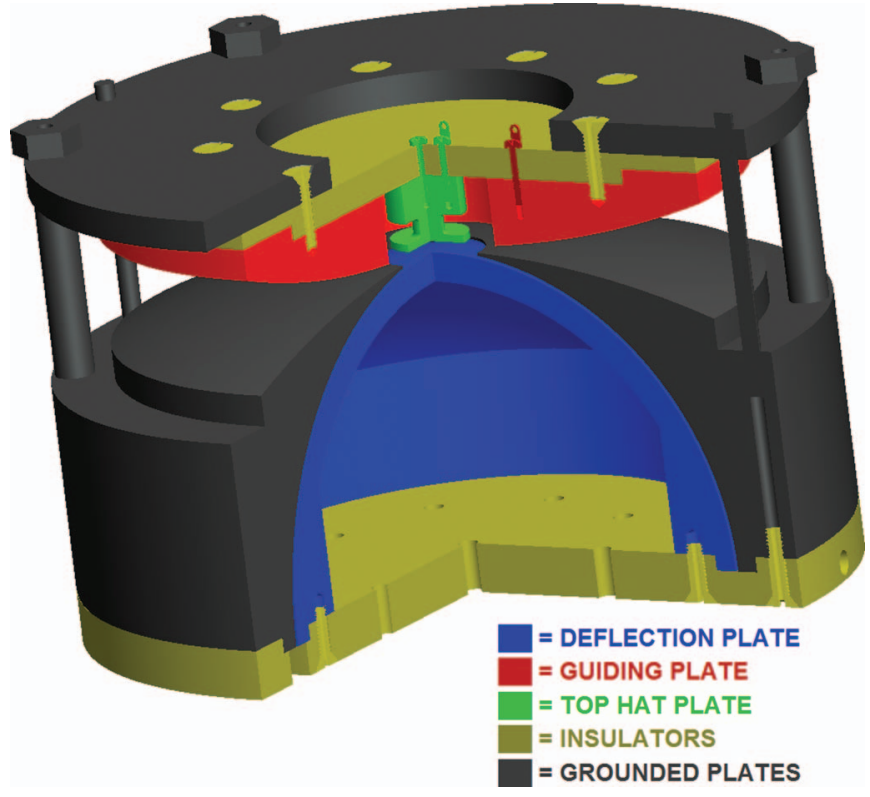

FIG. 4. (Color) Computer-aided design model of TOPAZ in PRO/ENGINEER.

enclosure is grounded, such that the potential field within the box is nearly zero and the trajectory of ions into the instrument remains undisturbed.

\section{CONSTRUCTION OF TOPAZ}

The biased and grounded plates in TOPAZ are made of Aluminum 6061-T6. Delrin ${ }^{\circledR}$ and Glass-Mica insulators are used to position the aluminum plates of TOPAZ in the correct position. Delrin ${ }^{\circledR}$ is used as a spacer between the top cover grounded plate and biased guiding and top hat plates. Glass-Mica, a ceramic with low thermal expansion, provides the required separation to create the gap distance between the deflection plate and grounded plate. Figure 4 displays a PRO/ENGINEER model of TOPAZ with the aluminum and insulator plates, screws, and added structural support members.

Since all of the plates and insulators of TOPAZ are cylindrically symmetric, a high tolerance Romi M17 CNC lathe, which is accurate to within 0.002 in. $(0.05 \mathrm{~mm})$, provided a great resource for the construction of all the parts. Figure 5 is the completed construction of TOPAZ.

\section{ANALYZER CALIBRATION}

To fully characterize the performance of TOPAZ, both SIMION computational results and experimental measurements conducted with an ion-beam accelerator are compared.

\section{A. Computational setup}

Every solution to the Laplace equation [Eq. (8)] is directly scalable since the Laplacian is a linear operator. The potential $\varphi$ at every point can be multiplied by a constant yielding another solution to a new set of plate potentials, as long as the ratio between the plate potentials remains constant. For the simulations run on TOPAZ, the deflection plate potential was set to $-300 \mathrm{~V}$. Equation (2) dictates that $15 \mathrm{keV}$ singularly charged ions should be measured at this plate potential. The ratios of the guiding plate and top hat

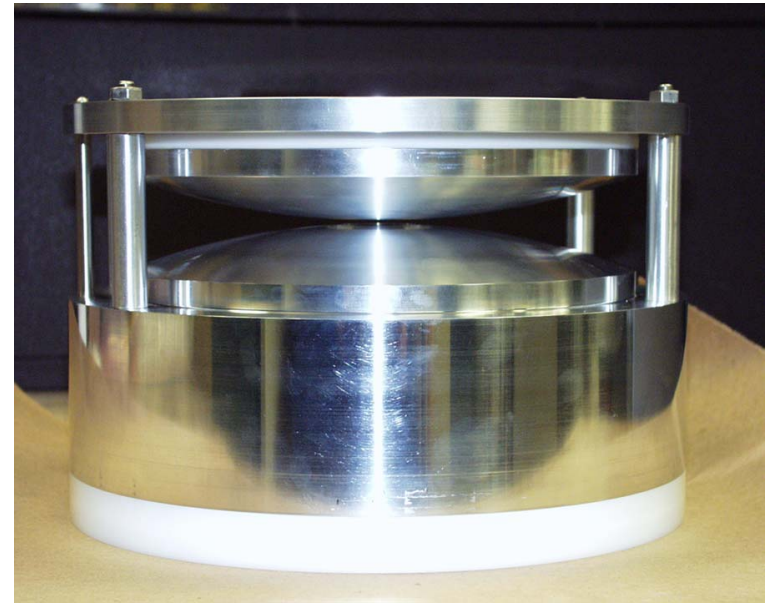

FIG. 5. (Color online) Final construction of TOPAZ.

plate potentials were varied with respect to the deflection plate to computationally characterize TOPAZ.

For each simulation over $1 \times 10^{6}$ ions were flown towards TOPAZ with varying velocities, elevation angles, and positions to cover the entire entrance aperture. Each of the ion initial angles and velocities in each direction were recorded if it made it to the exit of the gap (i.e., to the collector). Distributions of these properties were created to determine what type of ions TOPAZ detected for a specific plate setting.

\section{B. Ion beam and channel electron multiplier}

The ion-beam accelerator system, provided by the Department of Atmospheric, Oceanic, and Space Sciences at the University of Michigan, was employed to calibrate TOPAZ. This facility consists of a Colutron model G-1 ion gun system attached to a $200000 \mathrm{~cm}^{3}$ cylindrical chamber and is capable of maintaining a base pressure of $10^{-6}$ Torr. The ion beam has an energy range from 500 to $30 \mathrm{keV}$ with a current up to $1.5 \mathrm{nA}$ over a 2-cm-diameter beam size $\left(0.477 \mathrm{nA} / \mathrm{cm}^{2}\right)$.

A model 30052 Channeltron ${ }^{\circledR}$ by Burle Electro-Optics with an aperture diameter of $0.82 \mathrm{~cm}$ was used to detect the ions exiting from TOPAZ through the gap. The channel electron multiplier (CEM) detects ions by accelerating them into a highly emissive secondary electron surface. An avalanche effect is created where these electrons create more secondary electrons, and a measurable pulse is created. An Agilent 53131A frequency counter measured the pulse frequency and provided a good estimate of the ion flux out of the exit of the gap.

\section{Gap uniformity}

The gap distance between the deflection plate and grounded plate must be relatively uniform to ensure the transmission of ions through all azimuthal angles $\beta$. TOPAZ was placed on a rotational stage capable of rotating TOPAZ about its centerline to within $0.001^{\circ}$ while the CEM remained underneath TOPAZ at the gap exit. This configuration allowed for beam ions to be measured through different azimuthal angles of the gap. 


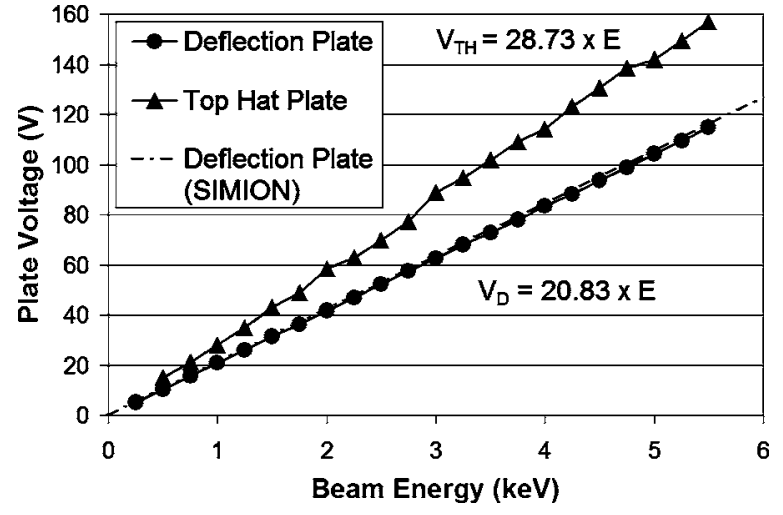

FIG. 6. Optimum deflection and top hat plate voltages as a function of beam energy.

Two test cases are presented: keeping the top hat plate voltage constant and optimizing the top hat plate voltage to maximize the counts. In both cases the deflection plate voltage was held constant. For a constant top hat plate voltage, a dip in the counts was seen for lower angles. This dip was attributed to a slight tilt relative to the axis of rotation, for which TOPAZ is highly sensitive to. If the top hat plate voltage was changed to maximize the number of counts, the transmission of ions was roughly constant from $-55^{\circ}$ to $57^{\circ}$. The decrease in transmission beyond these angles was due to the Delrin ${ }^{\circledR}$ plate base, which has a $120^{\circ}$ slot (from $-60^{\circ}$ to $60^{\circ}$ ) for the gap. Near the edges, fringe effects decrease the transmission of ions through the channel.

\section{Deflection plate response}

The ion accelerator provided an easy method of determining the deflection plate and energy-per-charge correlation. Optimum helping and deflection plate voltages could be determined over a range of beam energies. Figure 6 displays the measured and simulated relationship. A virtually linear relation exists between the two plate potentials and beam energy. The measured proportionality constant for the deflection plate differed from the SIMION computational results by $1.6 \%$, confirming the accuracy of the SIMION model.

\section{E. Guiding plate tests}

To correlate the guiding plate voltages with the measured particle elevation angles, a separate setup from the above tests was used. TOPAZ was mounted sideways on the rotational stage to emulate particles entering into the instrument from different elevation angles. Figure 7 presents the setup of TOPAZ for the guiding plate tests.

As TOPAZ was rotated about the aperture, ions enter at various elevation angles relative to the instrument. Rotating TOPAZ allowed for characterization of the guiding plates that steer ions in the vertical direction into the aperture. For each guiding plate voltage, the elevation angle with the most number of counts was found. These data show that a linear correlation between the guiding plate potential and elevation angle exists, a relation that was predicted by SIMION.

Figure 8 describes the guiding plate trends with the elevation angle. The measured counts and SIMION results were very similar. A more linear trend was measured with TOPAZ

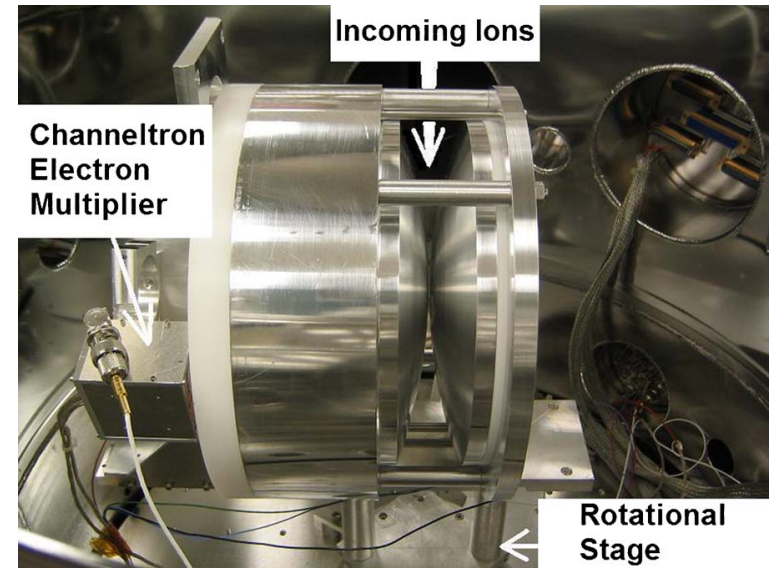

FIG. 7. (Color online) Guiding plate test setup inside the vacuum chamber.

than with the SIMION. Since the field generated by the guiding plate was over a larger distance, positive and negative voltages up to three times larger than the deflection plate voltage were required to retrieve ions from the full vertical angular field of view. The elevation field of view for TOPAZ was measured to be approximately $\pm 13^{\circ}$ since a sharp decrease in counts was seen beyond these angles.

\section{F. Resolution measurements}

Although the correlation between the biased plate voltages and particle angles and energies had been determined, the accuracy (or resolution) of each parameter is also important. By keeping the plate voltages on TOPAZ constant, the energy resolution was measured by tracking the transmission of ions as a function of the beam energy. The deflection plate potential was held constant, while the energy of the beam ions was varied. A distribution of detected counts as a function of the ion energy was determined. The equivalent was done through SIMION by profiling the energy distribution of ions that traveled through the gap. An example energy distribution at $3 \mathrm{keV}$ is displayed in Fig. 9. The measured and SIMION-deduced energy resolutions based on the full width at half maximum (FWHM) were $2.0 \%$ and $2.7 \%$, respectively, for this energy.

In a similar manner, the elevation angular resolution was determined by Monte Carlo simulation and by slightly ad-

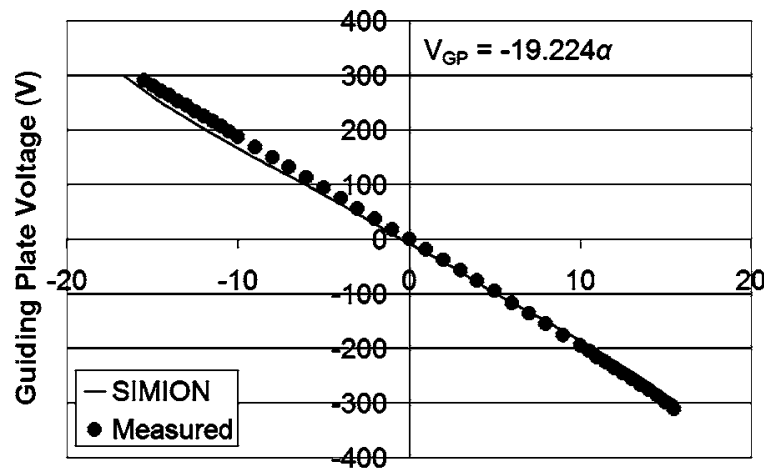

Elevation Angle $\alpha$ (degrees)

FIG. 8. Optimum guiding plate voltage as a function of elevation angle for a $5 \mathrm{keV}$ beam. A positive elevation angle corresponds to a particle entering TOPAZ from above the aperture. 


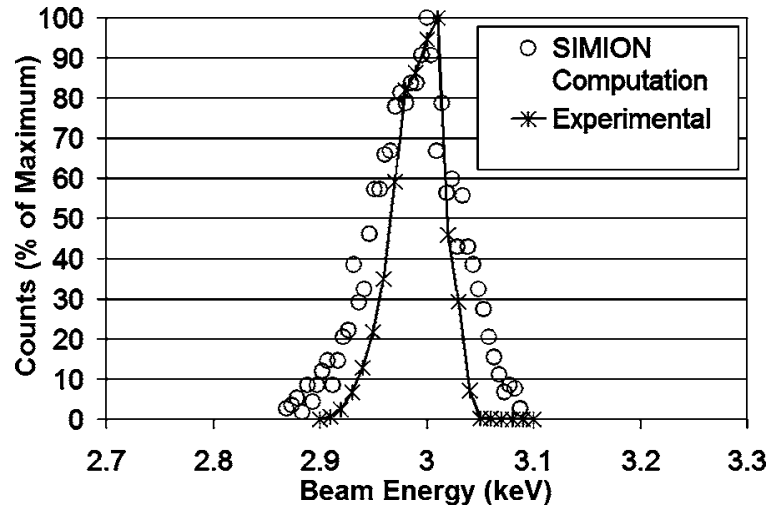

FIG. 9. Energy distribution for $3 \mathrm{keV}$ plate setting.

justing the elevation angle of the ions entering into TOPAZ with a specific guiding plate voltage. The elevation angle resolution is approximately $2^{\circ}$ according to both the guiding plate tests and SIMION results. The resolution for the azimuthal angle is a property of the ion focusing. SIMION suggests the ions to focus within $1^{\circ}$ at the exit of the gap, but this was never experimentally verified.

\section{G. Measurements on the NASA-173Mv1 Hall thruster}

TOPAZ was placed $1 \mathrm{~m}$ downstream of the NASA$173 \mathrm{Mv} 1$ Hall thruster in the Large Vacuum Testing Facility (LVTF) at the Plasmadynamics and Electric Propulsion Laboratory (PEPL). The thruster was operated at a discharge voltage of $300 \mathrm{~V}$ and discharge current of $5 \mathrm{~A}$. Two out of seven existing cryopumps were used to yield a purposely elevated pressure of $2.5 \times 10^{-5}$ Torr (corrected for xenon). At this pressure, the Debye shielding was highest, and the mean free path of the plasma ions are the lowest for test conditions on this thruster.

Measurements of the NASA-173Mv1 Hall thruster operating at an anode voltage of $300 \mathrm{~V}$ and current of 5 A displayed an energy-per-charge profile with a peak at $270 \mathrm{eV}$ (Fig. 10). The measurement of ions above the acceleration voltage is evidence of elastic collisions between singularly and doubly charged ions. This behavior has been observed in the similar power-level class P5 Hall thruster and the SPT100 at PEPL by Gulczinski et al. and King et al., respectively. ${ }^{14,15}$ The preliminary measurement by TOPAZ

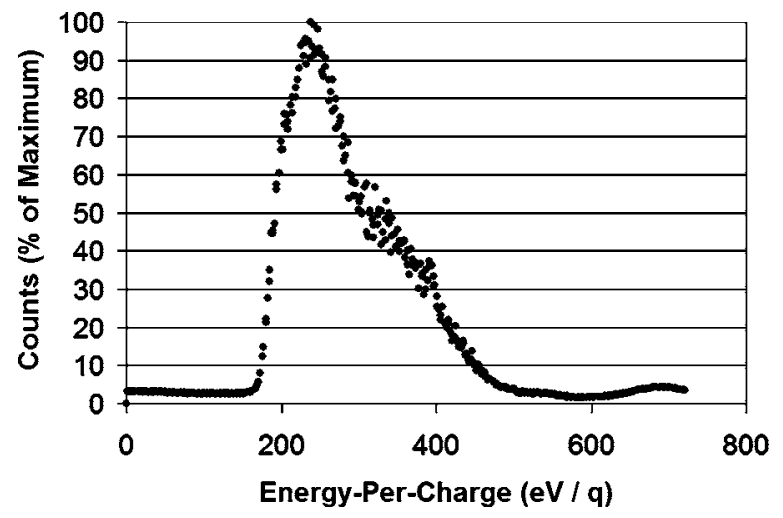

FIG. 10. Energy profile measured by TOPAZ for the NASA-173Mv1 Hall thruster operating at $300 \mathrm{~V}$ and $5 \mathrm{~A}$. confirms its successful operation as an energy analysis tool for Hall thruster plume diagnostics in a relatively high neutral density environment where facility effects can interfere with energy profile measurements.

\section{DISCUSSION}

A top hat electrostatic analyzer has been designed, created, and tested for Hall thruster plume studies. SIMION provided an accurate prediction of the analyzer's response towards a flux of beam ions under a vacuum. The ion-beam accelerator allowed for experimental calibration and characterization of TOPAZ. The design requirements for energy and angular resolutions were all met or exceeded. The vertical field of view $\left( \pm 13^{\circ}\right)$ was slightly less than anticipated $\left( \pm 15^{\circ}\right)$, and the azimuthal field of view was $112^{\circ}$ since structural constraints prevented taking advantage of the $360^{\circ}$ symmetry. Some discrepancies existed between the SIMION results and ion-beam measurements; however, an uncovered top hat plate voltage correlation with the elevation angle is a plausible explanation for this difference. Measurements on the NASA-173Mv1 demonstrated the operation of TOPAZ within the plasma environment of a Hall thruster plume.

\section{ACKNOWLEDGMENTS}

The authors greatly acknowledge Dr. Patrick Koehn for his endless support and lease of the ion accelerator, channel electron multiplier, and vacuum chamber for the characterization of TOPAZ. The authors thank Jessica Brooks for her SIMION simulation support and Chuck Navarre and Robb Gillespie for their highly precise machining of the parts. Finally, the authors thank Robert Lundgren for offering his expertise on the design of TOPAZ. One of the authors (A.L.V.) is supported by the NASA-funded Michigan Space Grant Consortium as well as the Department of Aerospace Engineering at the University of Michigan.

${ }^{1}$ P. Y. Peterson, D. T. Jacobson, D. H. Manzella, and J. W. John, AIAA Paper No. AIAA-2005-4243, 2005.

${ }^{2}$ D. Manzella, R. Jankovsky, and R. Hofer, AIAA Paper No. AIAA-20023676, 2002.

${ }^{3}$ D. T. Jacobson, R. S. Jankovsky, V. K. Rawlin, and D. H. Manzella, AIAA Paper No. AIAA2001-3777, 2001.

${ }^{4}$ A. D. Gallimore, J. Spacecr. Rockets 38, 441 (2001).

${ }^{5}$ S. J. Bame, D. J. McComas, D. T. Young, and R. D. Belian, Rev. Sci. Instrum. 57, 1711 (1986).

${ }^{6}$ J. H. Vilppola, P. J. Tanskanen, H. Huomo, and B. L. Barraclough, Rev. Sci. Instrum. 67, 1494 (1996).

${ }^{7}$ M. D. Rayman and D. H. Lehman, Acta Astronaut. 41, 289 (1997).

${ }^{8}$ J. E. Nordholt, D. T. Young, and H. O. Funsten, Aerospace Conference Proceedings, Big Sky, MT, Paper No. 10.1109/AERO. 2000.879327.

${ }^{9}$ M. L. R. Walker, R. R. Hofer, and A. D. Gallimore, AIAA Paper No. AIAA-2002-4253, 2005.

${ }^{10}$ D. A. Dahl, SIMION 3D Version 7.0 User's Manual (Idaho National Engineering and Environmental Laboratory, Idaho Falls, ID, 2000).

${ }^{11}$ R. H. Shih, Parametric Modeling with Pro/ENGINEER Wildfire (SDC, 2003).

${ }^{12}$ B. A. Banks, J. R. Gaier, C.-C. Hung, P. A. Walters, E. Sechkar, S. Panko, and C. A. Karnioti, AIAA Paper No. AIAA-2004-3815.

${ }^{13}$ C. W. Carlson and J. P. McFadden, Geophys. Monogr. 102, 125 (1998).

${ }^{14}$ F. S. Gulczinski, R. R. Hofer, and A. D. Gallimore, AIAA Paper No. AIAA-99-2430, 1999.

${ }^{15}$ F. S. Gulczinski and A. D. Gallimore, J. Propul. Power 17, 418 (2001). 